\title{
AS CONSEQUÊNCIAS DA ANDROPAUSA NA QUALIDADE DE VIDA: REVISÃO SISTEMÁTICA
}

Carolina Yamashita de MELLO ${ }^{1 *}$

Beatriz Santos ZÁCCARI²

Eugênia Mariel SAIKI ${ }^{3}$

Luiz Henrique Ledesma PEREIRA ${ }^{4}$

Laura Ferreira de REZENDE 5

\author{
${ }^{1-3}$ Discentes \\ ${ }^{4}$ Médico Urologista e Docente do curso de Medicina da UNIFAE. urolumi@terra.com \\ ${ }^{5}$ Mestre e Doutora pela Faculdade de Ciências Médicas UNICAMP e pós-doutora pela UNESP.laura@ fae.br \\ *carolinamello13@hotmail.com
}

Recebido em: 23/11/2016 - Aprovado em: 21/11/2017 - Disponibilizado em: 30/12/2017

\begin{abstract}
RESUMO
A Qualidade de vida está relacionada ao grau de satisfação encontrado na vida familiar, sexual, social, ambiental e à própria estética do indivíduo. Desenvolveu-se um estudo de revisão sistemática correlacionando a influência da andropausa ou distúrbio androgênico do envelhecimento masculino (DAEM), que consiste no decréscimo da produção de testosterona, na qualidade de vida dos homens acima de 40 anos, pois, nas duas últimas décadas tem se assistido à configuração de um novo modelo de representações acerca do envelhecimento masculino privilegia a dimensão do sexo, o qual de acordo com a Organização Mundial de Saúde (OMS) é considerado parâmetro para definir qualidade de vida. O trabalho em questão foi feito mediante a seleção e análise de artigos científicos coerentes com o tema, os quais foram encontrados nas bases de dados PubMed, Lilacs e Scielo. Desse modo, foram observados as consequências do Hipogonadismo de Início Tardio no estilo de vida do homem e os efeitos da reposição hormonal em indivíduos que sofrem dessa deficiência hormonal.
\end{abstract}

Palavras-chave: andropausa; qualidade de vida; hipogonadismo; estilo de vida; reposição hormonal.

\section{THE CONSEQUENCES OF ANDROPAUSE ON QUALITY OF LIFE}

\begin{abstract}
The quality of life is related to the degree of satisfaction found in family, sexual, social, environmental life and the individual's own aesthetic. A systematic review was conducted to correlate the influence of andropause or androgenic aging disorder (DAEM), which consists of a decrease in the production of testosterone, in the quality of life of men over 40 years of age, since in the last two decades it has If we assisted in the configuration of a new model of representations about male aging, it emphasizes the gender dimension, which according to the World Health Organization (WHO) is considered a parameter to define quality of life. The work in question was made through the selection and analysis of scientific articles consistent with the theme, which were found in PubMed, Lilacs and Scielo databases. Therefore, we observed the consequences of late-onset hypogonadism in the man's lifestyle and the effects of hormone replacement in individuals suffering from this hormonal deficiency
\end{abstract}

Keywords: andropause;quality of life;hypogonadism;style of life;hormonal replacement

\section{Introdução}

Andropausa, ou deficiência androgênica do envelhecimento masculino (DAEM), descreve a entidade clínica definida como uma síndrome associada ao envelhecimento. É uma condição frequente, mas

subdiagnosticada. $\mathrm{O}$ envelhecimento masculino possui sinais e sintomas semelhantes a deficiência androgênica em jovens e adultos, sendo esses, perda de 
energia, diminuição da libido, disfunção erétil, depressão, diminuição da massa e força muscular, osteoporose e osteopenia (Martits et al, 2004). Assim, é necessário diferenciar os efeitos fisiológicos e patológicos (Liberman et al, 2010).

Os níveis de testosterona em homens com idade compreendida entre 40 anos estão reduzidos, havendo uma diminuição anual de 0,3\%. Homens de 75 anos de idade possuem em média $66 \%$ da quantidade de testosterona que homens entre 25 anos possuem (Bonaccorsi et al, 2001).

A diminuição dos níveis de testosterona com envelhecimento, por um lado, está relacionada com a disfunção dos testículos (as células de Leydig sofrem redução de massa e uma diminuição no desempenho das células restantes, assim como redução da circulação sanguínea dos testículos). Porém, a redução dos hormônios estimuladores de testosterona, incluindo GnRH, a partir do hipotálamo e gonadotropina a partir da glândula pituitária.

$\mathrm{O}$ tratamento com testosterona em pacientes do sexo masculino de idade superior a 40 anos com andropausa irá elevar os níveis desse hormônio e também a qualidade de vida, a ereção, os níveis de energia, o desejo sexual, além da diminuição do índice de depressão (Rohden et al, 2011).

Portanto, o objetivo desse artigo é relacionar a influência da andropausa na qualidade de vida dos homens com idade superior a 40 anos.

\section{Método}

A revisão sistemática de literatura realizada nesse estudo utilizou as bases de dados Scielo, Pubmed e Lilacs.

Foi realizado pesquisa nas bases de dados, separadamente, utilizando os seguintes descritores: andropausa, hipogonadismo, qualidade de vida, em inglês e português.

Os critérios de inclusão foram: indivíduos do sexo masculino com idade superior a 40 anos. O critério de exclusão foi artigos publicados a partir de 2011 e artigos que associem a andropausa a outras doenças.

Inicialmente, cruzando os descritores nas 3 bases de dados escolhidas, foram encontrados 511 artigos. Destes, utilizando os critérios de inclusão e exclusão, foram selecionados ao todo 6 artigos.

(Anexo 1)

\section{Resultados}

Através dessa revisão sistemática, foram obtidos 6 artigos, os quais seguem abaixo:

(Anexo 2)

\section{Discussão}

Nos estudos de Almehmadi et al (2016), o tratamento com undecanoato de testosterona proporcionou uma melhora na função erétil 
após 3 semanas. No entanto, para resultados máximos, pode levar até 12 meses, demonstrando que o tratamento a longo prazo é necessário para alcançar ótimos efeitos. Assim, esse tratamento demonstrou que a testosterona melhora significativamente a função sexual, vitalidade, dor física, saúde geral e a qualidade de vida relacionada à saúde global em homens com andropausa, melhoras também demonstradas no estudo de Almehmadi et al (2016).

Dentre numerosos estudos, o estudo de Heidari et al (2014) demonstraram que $64 \%$ dos pacientes apresentavam irritabilidade, $77 \%$ apresentavam qualidade de vida insatisfatória e 12,5\% apresentavam sinais físicos de hipogonadismo. Todos os pacientes apresentavam tamanho normal de testículo e firmeza, enquanto $10 \%$ apresentavam micropene. Após o tratamento com uma única injeção intramuscular de $250 \mathrm{mg}$ de testosterona, $94 \%$ ficaram satisfeitos com sua libido. Além disso, outros sintomas incluindo perda de energia, qualidade de vida e irritabilidade foram resolvidos significativamente.

Além disso, no estudo de Sofimajidpour et al (2015), também foi observado que a administração oral de testosterona aumentou os níveis em homens com mais de 50 anos que estavam sofrendo de andropausa, sendo que a injeção intramuscular de testosterona teve um impacto maior que o uso oral. Os resultados benéficos constatados foram o aumento do número de ereções noturnas, melhora na função erétil e na satisfação sexual geral em homens com andropausa.

Além do mais, foi sugerido que a testosterona pode regular os mecanismos centrais da ereção peniana, bem como mecanismos neurais periféricos e vasculares. Além disso, melhorou a velocidade sistólica de pico e também diminuiu significativamente a velocidade diastólica final em homens com Hipogonadismo de Início Tardio.

Em contraste, este estudo também apresentou que a reposição hormonal aumentou a acne, a pele oleosa, a ginecomastia, o aceleramento do câncer de próstata para quem possui predisposição genética, doenças cardiovasculares e alterações lipídicas, além de aumentar os níveis de hematócrito.

Já segundo Canguven et al (2016), a terapia com uso de testosterona em homens com hipogonadismo de início tardio resultou em melhoria no fluxo sanguíneo das artérias cavernosas e na tumescência peniana noturna.

De acordo com Nigro et al (2012), a diminuição dos níveis de testosterona está associada aos sintomas depressivos e à fraca função cognitiva e à doença de Alzheimer. Estudos experimentais sugerem que a testosterona tem efeitos neuroprotetores. No entanto, em estudos de intervenção incluindo homens idosos, a reposição de testosterona só teve um efeito benéfico sobre o humor, em 
homens com níveis de testosterona claramente subnormal e é importante ter em mente que a disfunção sexual pode ter um grande impacto na qualidade de vida e psicossocial e bemestar emocional. Vários benefícios da terapia de reposição de testosterona em homens idosos foram observados, especialmente um aumento na massa muscular e densidade óssea, e uma diminuição na massa gorda, com dados mais conflitantes e controversos sobre força muscular, função sexual e humor.

Porém, o tratamento com testosterona pode acelerar o câncer de próstata metastático.

Em suma, foi considerado que a andropausa gera diversas consequências negativas ao indivíduo, sendo a reposição hormonal um possível método de tratamento, possuindo fatores positivos e negativos.

\section{Conclusão}

Através dos artigos revisados, este estudo demonstrou que a andropausa afeta a qualidade de vida dos homens de diversas formas, como foi relatado nos resultados.

Além disso, observa-se que a reposição de testosterona é um método a ser utilizado nessa deficiência.

Anexo 1 


\section{Anexo 1}

Número de relatos identificados no BD de buscas: 511

Lilacs: 7

Pubmed: 498

Scielo: 6

Número de relatos após eliminar os duplicados: 30

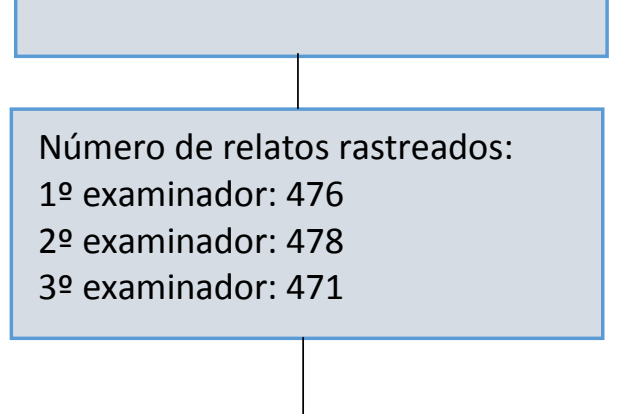

Número de artigos em texto completo avaliados para elegibilidade: 6

Número de artigos em texto completo excluído com justificativa: 34
Número de relatos excluídos

$1^{\circ}$ examinador: 35

$2^{\circ}$ examinador: 33

$3^{\circ}$ examinador: 40 


\section{Anexo 02}

Tabela I - Descrição dos artigos selecionados nas bases de dados PubMed, Lilacs e Scielo, quanto aos autores, ao desenho de estudo, métodos, resultados e explicações para as inferências.

\begin{tabular}{|c|c|c|c|c|}
\hline Autor/ano & Método & Avaliação & Resultados encontrados & $\begin{array}{l}\text { Possível explicação para os resultados } \\
\text { encontrados }\end{array}$ \\
\hline $\begin{array}{l}\text { Sofimajidpour et al, } \\
2015\end{array}$ & $\begin{array}{l}\text { Estudo quantitativo, } 80 \\
\text { homens em média entre } 51 \\
\text { e } 67 \text { anos, tratados com } \\
\text { cápsulas Testocaps } \\
\text { fabricadas por Schering- } \\
\text { Plough. }\end{array}$ & $\begin{array}{l}\text { Uso de questionário com } \\
\text { respostas de sim e não. }\end{array}$ & $\begin{array}{l}\text { Analisando o questionário } 100 \% \text { dos pacientes tinham } \\
\text { andropausa; após } 1 \text { mês de tratamento passou para } \\
87,5 \% \text {, no } 3^{\circ} \text { mês para } 55 \% \text { e após } 6 \text { meses } 0 \% \text {. } \\
\text { À medida que se avança a idade diminui-se o nível de } \\
\text { testosterona. } \\
\text { Os benefícios da terapia de testosterona foram } \\
\text { aumento da função sexual, densidade óssea, massa } \\
\text { muscular, melhora do humor, qualidade de vida e } \\
\text { função cognitiva, bem como aumento no poder. }\end{array}$ & $\begin{array}{l}\text { A administração oral de testosterona } \\
\text { aumentou os níveis em homens com mais } \\
\text { de } 50 \text { anos que estavam sofrendo de } \\
\text { andropausa. } \\
\text { A injeção intramuscular de testosterona } \\
\text { teve um impacto maior que o uso oral. }\end{array}$ \\
\hline $\begin{array}{l}\text { Canguven et al, } \\
2016\end{array}$ & $\begin{array}{l}\text { Estudo Prospectivo, } 88 \\
\text { homens com } \\
\text { hipogonadismo e } \\
\text { disfunção erétil, em média } \\
\text { de } 51,1 \text { anos. }\end{array}$ & $\begin{array}{l}\text { Uso de questionário para } \\
\text { investigar a extensão do } \\
\text { impacto da terapia com } \\
\text { uso de testosterona em } \\
\text { pacientes com } \\
\text { hipogonadismo em } \\
\text { termos de perfil clínico } \\
\text { andrológico. }\end{array}$ & $\begin{array}{l}\text { Uso de testosterona mostrou efeito benéfico para } \\
\text { função eréctil entre } 6 \text { e } 12 \text { meses de uso. Aumentos } \\
\text { significativos tanto na média de frequência e duração } \\
\text { da rigidez peniana, e melhoria do fluxo sanguíneo } \\
\text { arterial cavernoso. }\end{array}$ & $\begin{array}{l}\text { Terapia com uso de testosterona em } \\
\text { homens com LOH* resultou em melhoria } \\
\text { da função erétil, no fluxo sanguíneo das } \\
\text { artérias cavernosas e na tumescência } \\
\text { peniana noturna. }\end{array}$ \\
\hline $\begin{array}{l}\text { Almehmadi et al, } \\
2016 .\end{array}$ & $\begin{array}{l}\text { Foi realizado um ensaio } \\
\text { clínico com } 261 \text { pacientes } \\
\text { (idade média } 58 \text { anos) com } \\
\text { diagnóstico de } \mathrm{LOH}^{*} \\
\text { foram tratados com } \\
\text { undecanoato de } \\
\text { testosterona intramuscular } \\
\text { de ação prolongada por até } \\
5 \text { anos. }\end{array}$ & $\begin{array}{l}\text { Divisão entre } 2 \text { grupos } \\
\text { de comparação. }\end{array}$ & $\begin{array}{l}\text { Nos primeiros } 3 \text { meses, o Índice Internacional de } \\
\text { Sintomas da Próstata e os Sintomas de } \\
\text { Envelhecimento do Homem diminuíram } \\
\text { significativamente, sendo que o Índice Internacional } \\
\text { de Disfunção Erétil e testosterona total aumentaram. }\end{array}$ & $\begin{array}{l}\text { A testosterona é mostrada para melhorar } \\
\text { significativamente a função sexual e } \\
\text { vitalidade, dor física, saúde geral, e da } \\
\text { QVRS** global em homens com } \\
\text { deficiência de testosterona. }\end{array}$ \\
\hline
\end{tabular}




\begin{tabular}{|c|c|c|c|c|}
\hline Heidari et al, 2014. & $\begin{array}{l}\text { Pesquisa quantitativa com } \\
48 \text { pacientes do sexo } \\
\text { masculino com disfunção } \\
\text { erétil e sintomas } \\
\text { sugestivos de } \\
\text { LOH* com idade média de } \\
53 \text { anos. }\end{array}$ & $\begin{array}{l}\text { Uso de questionário e } \\
\text { histórico de condições } \\
\text { médicas baseado sobre as } \\
\text { declarações dos } \\
\text { pacientes. }\end{array}$ & $\begin{array}{l}\text { Um total de } 31(64 \%) \text { pacientes apresentaram } \\
\text { irritabilidade, } 37(77 \%) \text { apresentaram qualidade de } \\
\text { vida insatisfatória e seis }(12,5 \%) \text { apresentaram sinais } \\
\text { físicos de hipogonadismo. Todos os pacientes } \\
\text { apresentavam tamanho normal de testículo e firmeza, } \\
\text { enquanto cinco }(10 \%) \text { apresentavam micropênis. Após } \\
\text { o tratamento, } 45(94 \%) \text { pacientes estavam satisfeitos } \\
\text { com sua libido. Além disso, outros sintomas de LOH* } \\
\text { incluindo perda de energia, qualidade de vida e } \\
\text { irritabilidade resolvidos significativamente. }\end{array}$ & $\begin{array}{l}\text { Destaca-se que o nível sérico de } \\
\text { testosterona não aumentou } \\
\text { significativamente após o estudo }(\mathrm{P}= \\
0,997) \text {, o que pode ser explicado pela } \\
\text { natureza de curta duração da injeção } \\
\text { utilizada, com duração entre } 10 \text { e } 14 \text { dias. } \\
\text { No entanto, a redução significativa no } \\
\text { nível sérico de hormônio luteinizante após } \\
3 \text { semanas (P <0,001) indica a resposta } \\
\text { bioquímica à administração de } \\
\text { testosterona. }\end{array}$ \\
\hline Nigro et al, 2012. & $\begin{array}{l}\text { Ensaio clínico controlado } \\
\text { por placebo incluindo } 108 \\
\text { homens idosos com níveis } \\
\text { médios de testosterona de } \\
12,8 \text { Nmol. }\end{array}$ & $\begin{array}{l}\text { Divisão entre grupo } \\
\text { placebo e com uso de } \\
\text { testosterona }\end{array}$ & $\begin{array}{l}\text { Vários benefícios da terapia de reposição de } \\
\text { testosterona em idosos, como aumento da massa } \\
\text { muscular e densidade óssea, diminuição na massa } \\
\text { gorda e dados conflitantes e controversos sobre força } \\
\text { muscular, função sexual e humor. No entanto, a longo } \\
\text { prazo, riscos cardiovasculares e associados à próstata } \\
\text { permanecem desconhecidos. }\end{array}$ & $\begin{array}{l}\text { Os resultados de estudos controlados com } \\
\text { placebo em ensaios randomizados sobre os } \\
\text { efeitos da testosterona na qualidade de } \\
\text { vida e humor depressivo têm sido } \\
\text { inconsistentes e muitas vezes a qualidade } \\
\text { de vida avaliada por diferentes } \\
\text { questionários não melhorou } \\
\text { significativamente. }\end{array}$ \\
\hline Ros et al, 2012 & $\begin{array}{l}\text { Estudo prospectivo com } \\
125 \text { homens de idade } \\
\text { média de } 62 \text { anos com } \\
\text { baixo desejo sexual e } \\
\text { níveis de testosterona } \\
\text { abaixo de } 400 \mathrm{ng} / \mathrm{dL} \\
\text { foram selecionados para } \\
\text { receber CC } * * * \text {. }\end{array}$ & $\begin{array}{l}\text { Os pacientes foram } \\
\text { perguntados se eles } \\
\text { experimentaram } \\
\text { quaisquer efeitos } \\
\text { colaterais relacionados } \\
\text { com o uso de CC*** e se } \\
\text { eles experimentaram } \\
\text { qualquer melhoria no seu } \\
\text { perfil sexual. }\end{array}$ & $\begin{array}{l}\text { Os níveis séricos de testosterona variaram de } 309 \mathrm{ng} / \\
\mathrm{dL} \text { (valor médio de referência) a } 642 \mathrm{ng} / \mathrm{dL} \text { ( } 3 \text { meses } \\
\text { após iniciação de citrato de clomifeno, valor médio) (p } \\
<0,001 \text { ). }\end{array}$ & $\begin{array}{l}\text { O aumento médio do nível sérico de } \\
\text { testosterona em pacientes mais jovens foi } \\
\text { estatisticamente maior do que o aumento } \\
\text { médio de pacientes mais velhos. }\end{array}$ \\
\hline
\end{tabular}

* Hipogonadismo de Início Tardio; **Qualidade de Vida Relacionada à Saúde; ***Citrato de Comifeno 


\section{Referências}

BONACCORSI et al. Andropausa:

Insuficiência Androgênica Parcial do

Homem Idoso. Uma Revisão. Arq Bras

Endocrinol Metab. São Paulo, Brasil.

v.45 n.2, 2001.

JAKIEL et al. Andropause - state of the art and review of selected aspects.Prz

Menopauzalny. v. 14 n.1, p. 1-6, 2015.

LIBERMAN et al. Andropausa. RBM. São Paulo, Brasil. v. 68 n. 5. p. 150153, 2010.

MARTITS et al. Hipogonadismo masculino tardio ou andropausa. Rev. Assoc. Med, Bras, São Paulo. v.50 n. 4, 2004.

NORIEGA et al. La actitud y la Testosterona, la hormona del estilo de vida. Horiz Med. Lima, Peru. v. 2, p. $46-50,2013$.

ROHDEN et al. "O homem é mesmo a sua testosterona": promoção da andropausa e representações sobre sexualidade e envelhecimento no cenário brasileiro. Horizontes Antropológicos. Porto Alegre, Brasil. v 17, n. 35, p. 161-196, 2011.

SOFIMAJIDPOUR et al. The Effect of Testosterone on Men With Andropause. Iran Red Crescent Med J. v. 17. n. 12: e19406. 2015. 